This item is the archived peer-reviewed author-version of:

\title{
Direct observation of luminescent silver clusters confined in faujasite zeolites
}

\section{Reference:}

Altantzis Thomas, Coutino-Gonzalez Eduardo, Baekelant Wouter, Martinez Gerardo, Abakumov Artem M., Van Tendeloo Gustaaf, Roeffaers Maarten B.J., Bals Sara, Hofkens Johan.- Direct observation of luminescent silver clusters confined in faujasite zeolites

ACS nano - ISSN 1936-0851 - 10:8(2016), p. 7604-7611

Full text (Publisher's DOI): http://dx.doi.org/doi:10.1021/ACSNANO.6B02834

To cite this reference: http://hdl.handle.net/10067/1345760151162165141 


\section{Direct Observation of Luminescent Silver Clusters}

\section{Confined in Faujasite Zeolites.}

Thomas Altantzis, ${ }^{t, t}$ Eduardo Coutino-Gonzalez, ${ }^{\delta, t}$ Wouter Baekelant, ${ }^{\delta}$ Gerardo T. Martinez, ${ }^{\dagger}$

Artem M. Abakumov, ${ }^{\dagger, *}$ Gustaaf Van Tendeloo, ${ }^{\dagger}$ Maarten B.J. Roeffaers, ${ }^{\perp}$ Sara Bals,,${ }^{, *}$ and Johan Hofkens. ${ }^{\delta}{ }^{*}$

${ }^{\dagger}$ EMAT, University of Antwerp, Groenenborgerlaan 171, B-2020 Antwerpen, Belgium.

${ }^{\delta}$ Chem\&Tech - Molecular Imaging and Photonics, KU Leuven, Celestijnenlaan 200F, B-3001

Leuven, Belgium.

${ }^{\perp}$ Chem\&Tech - Centre for Surface Chemistry and Catalysis, KU Leuven, Celestijnenlaan 200F, B-3001 Leuven, Belgium.

KEYWORDS: HAADF-STEM, nanoporous materials, self-assembly, novel phosphors, Rietveld analysis. 


\section{ABSTRACT}

One of the ultimate goals in the study of metal clusters is the correlation between the atomicscale organization and their physicochemical properties. However, direct observation of the atomic organization of such minuscule metal clusters is heavily hindered by radiation damage imposed by the different characterization techniques. We present direct evidence of the structural arrangement, at an atomic level, of luminescent silver species stabilized in faujasite (FAU) zeolites using aberration corrected scanning transmission electron microscopy. Two different silver clusters were identified in Ag-FAU zeolites, a trinuclear silver species associated to green emission and a tetranuclear silver species related to yellow emission. By combining direct imaging with complementary information obtained from X-ray powder diffraction and Rietveld analysis, we were able to elucidate the main differences at an atomic scale between luminescent (heat-treated) and non-luminescent (cation-exchanged) Ag-FAU zeolites. It is expected that such insights will trigger the directed synthesis of functional metal nanoclusters-zeolite composites with tailored luminescent properties. 
Oligoatomic metal clusters with sub-nanometer dimensions have recently emerged as promising materials for application in diverse fields including catalysis, ${ }^{1,2}$ electronics, ${ }^{3}$ and photonics. ${ }^{4-7}$ Bare metal clusters tend to aggregate into larger nanoparticles, irreversibly losing their desired physicochemical properties. A solution to overcome this problem is the use of confining scaffolds to stabilize these sub-nanometer metal structures also referred to as metal nanodots. ${ }^{8,9}$ Due to their well-defined crystalline structure composed of pores, cages and cavities of molecular dimensions and due to the high cation exchange capacity, zeolites are promising scaffolds to stabilize such oligoatomic metal clusters. ${ }^{10-12}$ Representative examples are silverzeolite composite materials often employed as catalysts, ${ }^{13}$ adsorbents, ${ }^{14}$ molecular sieves,${ }^{15}$ and recently as highly luminescent composites with an emission spanning over the whole visible spectrum and external quantum efficiencies reported of up to $97 \% .{ }^{16-19}$ A detailed structural characterization of such oligoatomic silver clusters confined in zeolites at the atomic scale is crucial for understanding the size-structure function relationship and for rational material optimization. However, the direct structural characterization of such small silver nanodots or clusters comprising just a few atoms inside zeolite scaffolds is challenging due to the sensitivity of the silver clusters and zeolite framework to radiation damage (in situ restructuring, increased mobility) imposed by the different characterization techniques. ${ }^{20,21}$

X-ray crystallography has been extensively used to determine the position of metal ions and clusters as well as the structure of the latter embedded in crystalline matrices. ${ }^{22}$ This technique provides averaged information on the mean atom positions within the ordered framework structure. As such, X-ray diffraction (XRD) has been one of the preferred characterization techniques to study the location of inorganic/organic guests inside natural and synthetic zeolites, ${ }^{23}$ and in the case of silver exchanged zeolites, several models have been put forward for 
the most commonly used topologies (FAU, LTA). For instance, Gellens and co-workers ${ }^{24}$ found partially reduced linear $\mathrm{Ag}_{3}$ species located inside the hexagonal prisms of Ag-FAUX zeolites ( $\mathrm{Si} / \mathrm{Al}: 1.23)$. Lee and collaborators ${ }^{25}$ reported the formation of $\mathrm{Ag}_{2}{ }^{2+}, \mathrm{Ag}_{3}{ }^{+}, \mathrm{Ag}_{3}{ }^{2+}$ silver species in Ag-FAUX zeolites (Si/Al : 1.08) heat-treated under an oxygen flow. In a related study, Kim and co-workers observed the presence of larger silver species $\left(\mathrm{Ag}_{4}{ }^{\mathrm{n}+}, \mathrm{Ag}_{8}{ }^{\mathrm{m}+}\right)$ in fully exchanged Ag-FAUX zeolites (Si/Al : 1.08) exposed to a hydrogen flow. ${ }^{26}$ However, despite the many efforts carried out to decipher the exact structure of silver clusters in FAU zeolites, to date there is no conclusive model obtained.

Transmission electron microscopy (TEM) is a very powerful technique to characterize the structure and composition of materials at an atomic scale. However, zeolites and silver clusters are known to suffer from radiation damage. ${ }^{21,27,28}$ For zeolites this is especially the case for topologies having a relatively low $\mathrm{Si} / \mathrm{Al}$ ratio such as LTA and FAU zeolites. Recently, high angle annular dark field scanning transmission electron microscopy (HAADF-STEM) was used to unravel the structure of sub-nanometer silver species in fully exchanged Ag-LTA zeolites, ${ }^{21}$ which had been a matter of debate for over four decades. An octahedral $\mathrm{Ag}_{6}$ structure, surrounded by a cubic arrangement containing $8 \mathrm{Ag}$ atoms located at framework positions, was clearly visualized within the sodalite cages of heat-treated Ag-LTA zeolites. Nevertheless, the systematic analysis and correlation between the atomic scale organization and the photoluminescence was not addressed.

Here, we employed aberration corrected high angle annular dark field scanning transmission electron microscopy (HAADF-STEM) to investigate the atomic arrangement of luminescent Ag clusters in FAU zeolites. Due to the extreme sensitivity of Ag-zeolite materials to electron beam irradiation, we employed a relatively low electron dose. Additionally, the signal-to-noise ratio 
was significantly improved by statistically averaging of the HAADF-STEM images. By combining TEM results with powder XRD and subsequent Rietveld analysis, a complete 3D structural characterization of heat-treated luminescent silver exchanged FAU zeolites with different $\mathrm{Si} / \mathrm{Al}$ ratios could be achieved and the clusters from which the luminescence is originating were identified. Moreover, structural differences between the samples in their luminescent and non-luminescent states were also revealed.

\section{RESULTS AND DISCUSSION}

HAADF-STEM was used in this study to directly visualize Ag atoms confined in FAU zeolites. We combined monochromated aberration corrected HAADF-STEM, along the principal zone axes of the FAU zeolites, with a systematic image analysis in order to determine differences in the 3-dimensional positions of Ag atoms present in the face-centered cubic (fcc) crystalline structures of Ag-FAU zeolites. HAADF-STEM images acquired along the [100] and [110] zone axes were used to build the 3D models for Ag-FAU samples, and images along the [111] zone axis were used to confirm this model. Images along [111] of Ag-FAUX and Ag-FAUY are not included here, but two images along [111] are displayed, in the supplementary material, for the cation-exchanged (non-luminescent) forms of both Ag-FAUX and Ag-FAUY zeolites (Supporting Information, Figure S1).

Ag-FAUX. Figure 1 shows HAADF-STEM images of Ag-FAUX (cation-exchanged and heattreated), acquired along the [110] and [100] zone axes. It is of great importance to minimize the electron dose during the acquisition in order to avoid electron beam damage of the Ag-zeolite material during the data acquisition. Because of the low electron dose, the images exhibited a low signal-to-noise-ratio ( $\mathrm{S} / \mathrm{N}$ ratio). To overcome this problem, a template matching procedure 
was applied to the acquired data. This technique was used to find specific regions in a given image which correspond to a template. ${ }^{29}$ More details of the procedure can be found in the Supporting Information (section S-I and Figure S2). The result of this procedure is presented as an inset for each zone axis in Figure 1 . The $\mathrm{S} / \mathrm{N}$ ratio is indeed significantly increased in comparison to the raw data.

Because of the chemical sensitivity of the HAADF-STEM technique, it can be assumed that the bright spots in the insets correspond to the projected positions of the Ag atoms in the zeolitic framework. HAADF-STEM images yield an intensity which scales with the atomic number Z and the thickness of the sample. ${ }^{30}$ Therefore, Ag atoms will appear with higher intensity compared to the zeolitic framework elements ( $\mathrm{Si}, \mathrm{Al}$ and $\mathrm{O})$. From the template matched images (insets Figure 1) it is clear that the intensity of the bright spots is not equal. This can be explained by the presence of multiple Ag atoms along the same projected position in the HAADF-STEM image.

Due to the complexity of the HAADF-STEM image analysis, we performed complementary powder XRD measurements and Rietveld refinement of the crystal structures on the samples in their luminescent (heat-treated) and non-luminescent (cation-exchanged) states to assist the construction of the 3D structural models for Ag-FAUX samples. The combined results are displayed in Figure 2, where the HAADF-STEM images with improved S/N ratio are presented along with an overlay of the silver positions derived from XRD measurements. All XRD determined Ag positions have high occupancy factors (the occupancy factor is a measure of the fraction of molecules in a crystal in which an atom actually occupies the position specified in a proposed model) for both the cation-exchanged and the heat-treated samples (see Table 1). For both zone axes, a reasonable agreement was found between the positions of the Ag atoms 
determined by comparing the averaged HAADF-STEM images and occupancy factors obtained by XRD analysis.

Based on these Ag positions we developed a 3D structural model of Ag-FAUX zeolites in their luminescent (heat-treated) and non-luminescent (cation-exchanged) states. For the cationexchanged Ag-FAUX sample Ag atoms are predominantly present at sites I, I' and II (Supporting Information, Figure S5); whereas in the heat-treated sample sites I, I', II and II' are mainly populated (Table 1, Figure 3). This information suggests the presence of three adjacent Ag atoms linearly arranged at sites I'-I-I' at an interatomic distance of $3.129 \AA$ already before heattreatment. However, the occupancy factors also show the low probability for the actual formation of this linear $\left[\mathrm{Ag}_{3}\right]$ species (Supporting Information, Table S2). Additionally, site II (outer site of the six ring of sodalite cages, see Figure S5, Supporting Information) display a high silver atom occupancy (0.966) in the cation-exchanged samples. Nevertheless, this silver is located at a distance of $4.321 \AA$ ruling out significant interactions with the silver atoms at sites I' and I (Supporting Information, Table S5). Once the samples are heat-treated, site II' (inner site of the six ring of the sodalite cages, see Figure S5, Supporting Information) becomes populate. Based on the changes in occupancy factors, a migration of Ag from site II to II' takes place (indicated by blue arrows in Figure 3). Further, the interatomic distance between the silver atoms at sites I'I-I' slightly decreases $(3.123 \AA$ ) and judging from the occupancy factors, the probability for the formation of the linear $\left[\mathrm{Ag}_{3}\right]$ species increases (Supporting Information, Table S3). Based on these silver positions, the linear $\left[\mathrm{Ag}_{3}\right]$ silver species along the hexagonal prism connectors seem to weakly interact with Ag atoms located at position II' which are at $3.505 \AA \AA$ resulting in a tetranuclear $\left[\mathrm{Ag}_{3} \ldots \mathrm{Ag}\right]$ silver species (Figure 3). Such interaction might be originated from an autoreduction process in which silver cations are partially reduced by electrons coming from the 
oxidation of water to oxygen during heat-treatment, as suggested elsewhere, ${ }^{16}$ with the subsequent orbital interactions between silver atoms ${ }^{31}$ which could generate discrete energy levels and lead to electronic transitions resulting in intense light absorption and emission. ${ }^{32}$ This $\left[\mathrm{Ag}_{3} \ldots \mathrm{Ag}\right]$ silver species is likely to be responsible for the yellow emission in these heat-treated Ag-FAUX samples (Figure 3c,d,e). To further corroborate this theory, we exchanged FAUX zeolites with $\mathrm{Cu}^{2+}$ and $\mathrm{Ag}^{+}$ions and proceeded with their heat-treatment. It is well known that $\mathrm{Cu}$ (II) ions have a great affinity to occupy site I in FAU topologies. ${ }^{23}$ Therefore we expected a decrease of the luminescence of the heat-treated $\mathrm{Ag} / \mathrm{Cu}$-FAUX sample since the formation of the linear $\left[\mathrm{Ag}_{3}\right]$ will be hampered. The emission profiles of Ag-FAUX, $\mathrm{Ag} / \mathrm{Cu}-\mathrm{FAUX}$, and $\mathrm{Cu}-$ FAUX samples are displayed in Figure S7 (Supporting Information). A decrease of about three orders of magnitude in photoluminescence of $\mathrm{Ag} / \mathrm{Cu}-\mathrm{FAUX}$ samples was observed when compared to Ag-FAUX zeolites, supporting our theory.

Next, we analyzed the stoichiometry of the investigated samples. The FAUX zeolite used in this study has the following chemical composition; $\mathrm{Na}_{88}\left[\mathrm{Al}_{88} \mathrm{Si}_{104} \mathrm{O}_{384}\right]$ (per unit cell, Supporting Information, Table S6). Hence, based on charge compensation $88 \mathrm{Ag}(\mathrm{I})$ atoms can theoretically be accommodated within one FAUX unit cell. Nevertheless, the EDS analysis performed in AgFAUX samples showed the presence of only $78 \mathrm{Ag}$ atoms per unit cell. Furthermore, from the XRD analysis of the non-activated and activated Ag-FAUX samples only $66 \mathrm{Ag}$ atoms per unit cell were observed (Supporting Information, Table S3). This might indicate that the remaining $12 \mathrm{Ag}$ atoms are highly mobile, as suggested elsewhere, ${ }^{25}$ or are located at sites with very low occupancy factors (such as site III and III').

Ag-FAUY. The same experimental approach was followed for the structural characterization of the Ag-FAUY samples, for both the cation-exchanged (non-luminescent) and the heat-treated 
(luminescent) forms. The HAADF-STEM images together with the averaged results are given in Figure 4. HAADF-STEM images of Ag-FAUY samples along the [110] and [100] zone axes show no significant differences between the cation-exchanged and heat-treated samples. When carefully comparing the silver positions from the HAADF-STEM averaged images by template matching with the silver positions derived from XRD with Rietveld analysis (Figure 5) clearly inconsistencies emerged; the clearest differences are indicated by yellow circles in Figure 5 .

For the heat-treated sample, as illustrated in Figures $5 \mathrm{c}$ and $5 \mathrm{~d}$, these inconsistencies between STEM and XRD are prevalent at positions Ag1, Ag4 and Ag5 (cyan, yellow and blue atoms respectively, all located at site I'). Although Ag is observed at these positions by XRD on powdered samples, their presence cannot be confirmed in the STEM images. This discrepancy can be explained by the low occupancy of Ag at these positions (Table 2). For the cationexchanged sample, inconsistencies were found for the Ag1 position at site I' (cyan atoms), as indicated by yellow circles in Figures 5a and 5b. Averaged STEM images do not show bright intensities at this position even though high occupancy factors are derived from the XRD data.

Previous studies performed on partially and fully Ag-exchanged LTA and FAU zeolites, showed that applying high vacuum can have the same activating effect as heat treatment resulting in the formation of luminescent silver species. Some zeolite topologies like FAUY are more prone to this activation than others (e.g. FAUX). ${ }^{4,20,21,33,34}$ During TEM analysis, the samples were always kept inside the ultra-high vacuum of the column $\left(\sim 1 \times 10^{-7} \mathrm{mbar}\right)$ for at least 10 hours prior to the observations. Additionally, the samples were exposed to a highly energetic electron beam $(300 \mathrm{kV})$. Therefore it is very likely that the activation of the cationexchanged samples initially in the non-luminescent state has taken place during TEM investigation. In contrast, XRD measurements were done at ambient conditions and the radiation 
is much less intense and focused explaining the discrepancies observed between XRD and STEM data. To further investigate this issue, we conducted in-situ photoluminescence experiments on cation-exchanged Ag-FAUY zeolites using a vacuum compatible spectroscopic cell (Supporting Information, Figure S8). Cation-exchanged samples were pressed into pellets $(1.3 \mathrm{~cm}$ diameter and $1 \mathrm{~mm}$ thickness) and mounted into the cell after which they were evacuated and kept under vacuum conditions $\left(\sim 1 \times 10^{-5}\right.$ mbar $)$ overnight. Comparison of the luminescence before and after the vacuum treatment clearly shows a strong increase in luminescence similar to the typical heat activation (Supporting Information, Figure S9).

This explains why no differences could be observed between the Ag-FAUY samples in their non-luminescent (cation-exchanged) and luminescent (heat-treated) state in the HAADF-STEM images. Nevertheless, from XRD analysis we identified differences in the atomic arrangement of Ag in cation-exchanged and heat-treated Ag-FAUY samples. In sites I, I' and II, the presence of Ag atoms was observed in both the cation-exchanged and the heat-treated Ag-FAUY samples (Figure 6), however, with a clear difference in their occupancy factors (Table 2). $\mathrm{A}\left[\mathrm{Ag}_{3}\right]$ species with silver atoms located at positions I'-I-I', similar to FAUX, was elucidated from the XRD analysis (Figure 6). Based on the occupancy factors derived from XRD analysis the probability for the formation of such trinuclear species in the cation-exchanged sample is very low (Supporting Information, Table S4). In contrast in the heat-treated sample a delocalization of the $\mathrm{Ag}$ atoms positioned at site I' together with an increase of the occupancy factor of site I favors the formation of $\left[\mathrm{Ag}_{3}\right]$ species. Additionally, site II displays a very similar occupancy factor in both samples (cation-exchanged and heat-treated). In contrast to Ag-FAUX the heat treatment does not induce silver migration from site II to site II'. Therefore the green emission of heat- 
treated Ag-FAUY samples can be assigned to $\left[\mathrm{Ag}_{3}\right]$ species, interatomic distances for this species are displayed in the Supporting Information, Table S5.

Finally we performed a stoichiometric analysis of Ag-FAUY samples, based on the chemical composition the structural formula of this FAUY is $\mathrm{Na}_{52}\left[\mathrm{Al}_{52} \mathrm{Si}_{140} \mathrm{O}_{384}\right]$ (per unit cell, Supporting Information, Table S6). A maximum of $52 \mathrm{Ag}$ atoms can be exchanged per unit cell, assuming full cation replacement. Nevertheless, the EDS analysis performed on the samples revealed the presence of only $35 \mathrm{Ag}$ atoms per unit cell instead, a value that was also obtained from the XRD analysis of the cation-exchanged and heat-treated Ag-FAUY samples (Supporting Information, Table S4).

\section{CONCLUSIONS}

By using aberration corrected HAADF-STEM, at relatively low electron dose, assisted by powder XRD with Rietveld analysis, we were able to directly visualize for the first time the atomic arrangement of luminescent silver clusters in Ag-FAU zeolites possessing two different $\mathrm{Si} / \mathrm{Al}$ ratios. Surprising differences in organization of clusters and mobility of silver ions/atoms in very similar zeolite frameworks were revealed. The detailed atomic analysis of the samples in their luminescent state revealed the presence of a linear $\left[\mathrm{Ag}_{3}\right]$ clusters associated to green emission in Ag-FAUY zeolites, whereas in Ag-FAUX zeolites $\left[\mathrm{Ag}_{3} \ldots \mathrm{Ag}\right]$ clusters were observed and correlated to the typical yellow emission displayed by this sample. Remarkably, slight differences in cluster nuclearity and arrangement, at the atomic scale, have been shown to have a great impact on the photoluminescence features of silver clusters stabilized in FAU zeolites. Interestingly, both samples (Ag-FAUY and Ag-FAUX) share a common predecessor in their non-luminescent state, an apparent $\left[\mathrm{Ag}_{3}\right]$ species, which acts as a precursor for the subsequent 
formation of the luminescent Ag clusters in heat-treated Ag-FAU zeolites. The present report lays out a roadmap for studying the atomic scale organization of luminescent metal clusters confined in zeolites, and correlate such information to the luminescent properties of these clusters, enabling the rationalization and directed synthesis of functional metal-exchanged zeolites with tailored luminescent properties.

\section{METHODS}

Sample preparation. In this study, FAU zeolites (FAUX, Si/Al ratio 1.2 from UOP, and FAUY Si/Al ratio 2.7 from ZEOLYST) were employed (Supporting Information, Figure S4 and Table S6). A cation exchange procedure was used to generate the fully loaded silver exchanged FAU zeolites. In brief, one gram of zeolite material was suspended in $500 \mathrm{~mL}$ of an aqueous silver nitrate solution (10 mM, $\mathrm{AgNO}_{3}$ Sigma-Aldrich, $99.99 \%$ purity). The sample was left overnight in an end-over-end shaker for agitation in the dark. This procedure was repeated 4 times, using a fresh nitrate solution every time in order to drive cation replacement as far as possible to completion. Energy dispersive X-ray spectroscopy (EDS) carried out in a scanning electron microscope applied to the exchanged material showed $88 \%$ and $63 \%$ of cation exchange for Ag-FAUX and Ag-FAUY respectively. The samples were recovered by filtration using a Büchner filter and washed several times with miliQ water. The recovered powder was preheated at $373 \mathrm{~K}$ for 1 hour (cation-exchanged samples), to remove the excess water, and then transferred to a muffle for further calcination, first at $393 \mathrm{~K}$ for 1 hour and then until $723 \mathrm{~K}$ overnight (heat-treated samples), using a temperature ramp of $5 \mathrm{~K} / \mathrm{min}$. After calcination, the sample was cooled and stored in the dark. 
Powder XRD measurements. Silver exchanged FAU zeolites (Ag-FAUX and Ag-FAUY) contained in glass capillaries $(0.7 \mathrm{~mm}$ diameter $)$ heat-treated at 373 and $723 \mathrm{~K}$ were analyzed (Supporting Information, Figure S3). XRD patterns were recorded on a STOE STADI MP diffractometer with a linear position sensitive detector (PSD) $\left(6^{\circ} 2 \theta\right.$ window) in the region $2 \theta=$ 0 to $90^{\circ}$, with a step width of $0.5^{\circ}$, internal PSD resolution $0.01^{\circ}$, and a step time of $400 \mathrm{~s}$. The measurements were performed in Debye-Scherrer mode at room temperature using $\mathrm{CuK} \alpha_{1}$ radiation with $\lambda=1.54056 \AA$ selected by means of a Ge(111) monochromator. The Rietveld analysis was performed with the JANA2006 program. ${ }^{35}$ The crystallographic data on the silver exchanged zeolites from Gellens $e t a l .{ }^{24}$ were used as the initial model for the structure refinement. The refinement of the crystallographic positions constituting the framework was performed with the same atomic displacement parameter (ADP) as that reported elsewhere. ${ }^{24}$ The occupancy factors for the Ag positions were also refined with the joint ADP.

TEM sample preparation and set-up description. In order to avoid contact with air, the capillaries containing the zeolitic powder for each sample, as used for XRD measurements, were transferred to a glove box, where samples for TEM measurements were prepared by dispersing the zeolitic powder in anhydrous hexane. A droplet of the solution was deposited on a holey $\mathrm{C}$ coated $\mathrm{Cu}$ grid. The grid was placed in a Gatan model 648 double tilt vacuum transfer holder to avoid contact with air during the transfer of the sample into the TEM. Before TEM measurements, the sample was stored in the vacuum of the column $\left(\sim 1 \times 10^{-7} \mathrm{mbar}\right)$ for at least 10 hours. In this way we achieve a higher stability of the set-up during the measurements. High resolution HAADF-STEM images were acquired using an aberration corrected FEI Titan 50-80 transmission electron microscope operated at $300 \mathrm{kV}$. The probe convergence semi angle was set to $21 \mathrm{mrad}$ and the frame size for each acquisition was $1024 \times 1024$ pixels using a dwell time of 
$3 \mu$ s. Further exposure resulted into damage of the zeolite framework and the subsequent formation of larger silver nanoparticles as illustrated by the movie provided in the supporting information (Supporting Information, Video S1).

Photoluminescence characterization. For the luminescence excitation-emission characterization, the cation-exchanged and heat-treated Ag-FAU samples were placed in a quartz cuvette (1 mm path length) and sealed by a Teflon stopper. Emission and excitation spectra were recorded using an Edinburgh Instruments FLS 980 fluorimeter (corrected for wavelength dependence throughput and sensitivity of the detection channel). For every excitation wavelength (starting from 250 to $600 \mathrm{~nm}$ ), the emission spectrum was collected starting $30 \mathrm{~nm}$ shifted to the excitation wavelength up to $800 \mathrm{~nm}$ using $5 \mathrm{~nm}$ steps. The signal above $410 \mathrm{~nm}$ was measured using a $400 \mathrm{~nm}$ long pass filter to avoid interference from second-order excitation peaks; the measured intensities were corrected for the transmittance of the long pass filter. The emission was collected in "front face mode" through the quartz cuvette and sent to a monochromator and photomultiplier tube (PMT) for detection. From the separate emission spectra at varying excitation wavelengths, the two-dimensional excitation-emission matrices were constructed, the raw data were corrected for background and noise, and were interpolated to a resolution of $1 \mathrm{~nm}$ $\mathrm{x} 1 \mathrm{~nm}$. 

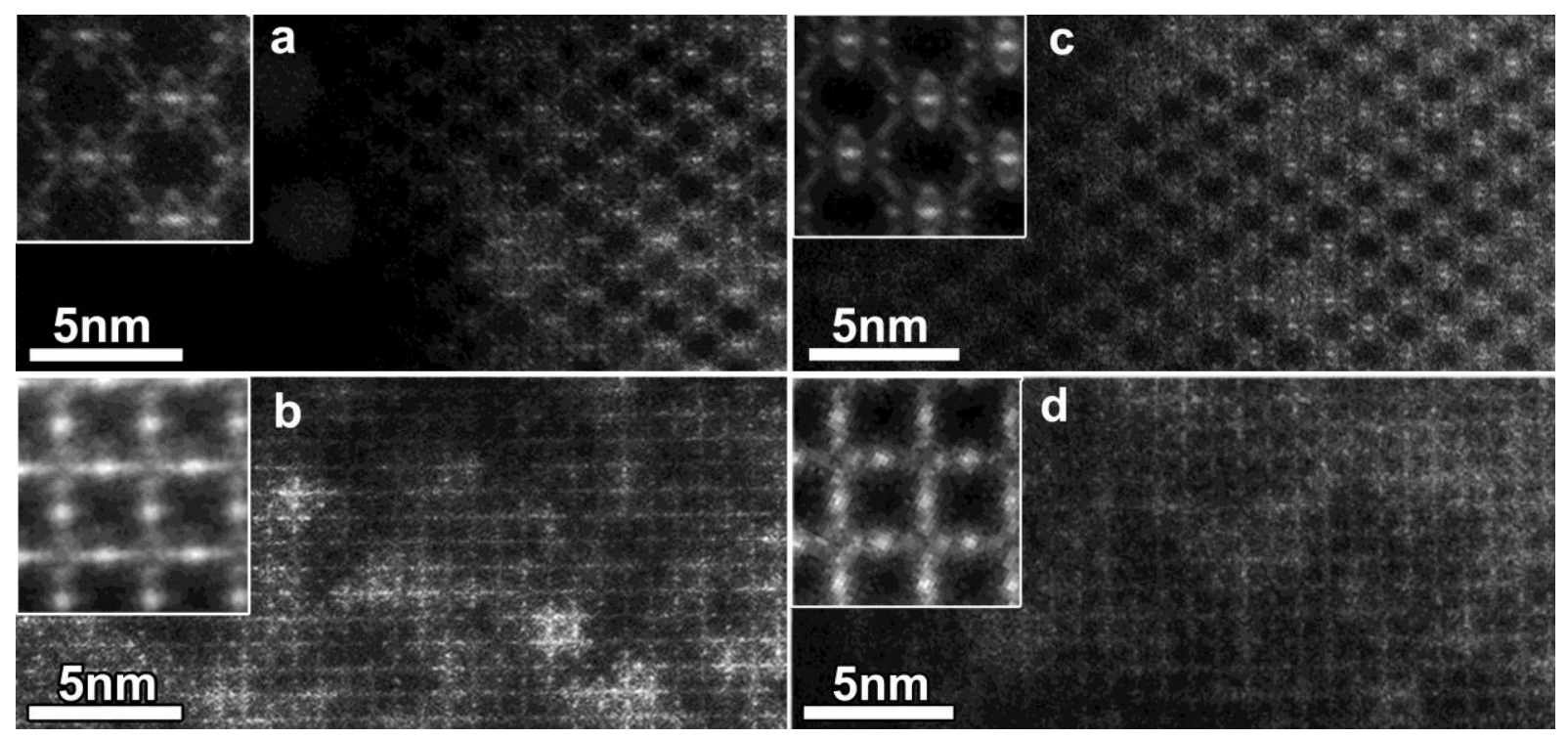

Figure 1. High resolution HAADF-STEM images of Ag-FAUX zeolites, acquired along a) [110] and b) [100] for the cation-exchanged sample and c) [110] and d) [100] for the heat-treated sample. The insets display the results of the averaging procedure, using 16 templates for (a), 161 for (b), 50 for (c) and 139 for (d), respectively. 

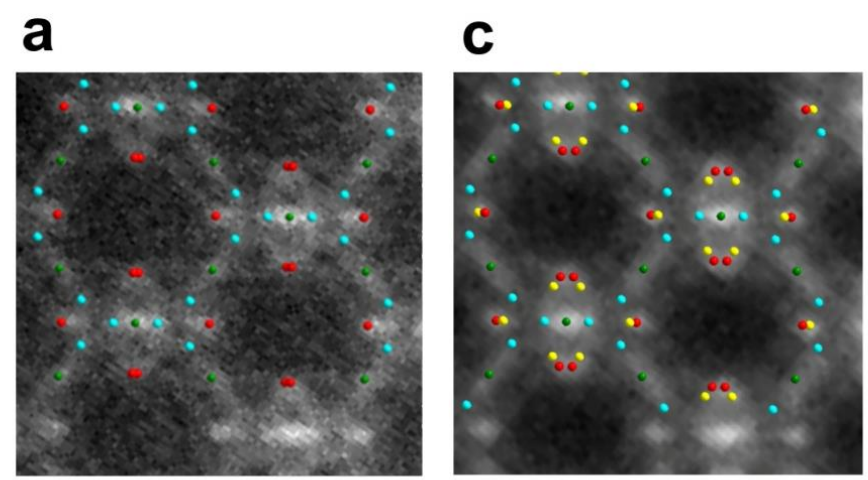

\section{$\underline{1 \mathrm{~nm}}$}

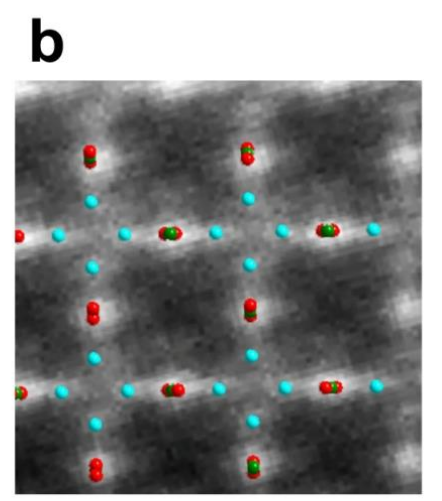

\section{d}

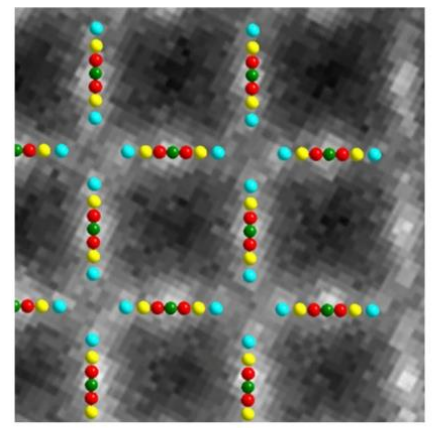

$1 \mathrm{~nm}$

Figure 2. a) and c) Overlay between the averaged HAADF-STEM images and the silver atomic positions obtained from XRD along the [110] zone axis for both the cation-exchanged (a) and heat-treated (c) forms of Ag-FAUX. b) and d) Overlay between the averaged HAADF-STEM images and the silver atomic positions obtained from XRD along the [100] zone axis for both the cation-exchanged (b) and heat-treated (d) forms of Ag-FAUX. The spheres with different colors represent the Ag atoms with different occupancy factors, which are given in Table 1. It can be seen that for both zone axis a very good matching is prevalent when comparing the averaged images with the models obtained from XRD analysis. For a better visualization, the zeolite framework atoms are not displayed. 

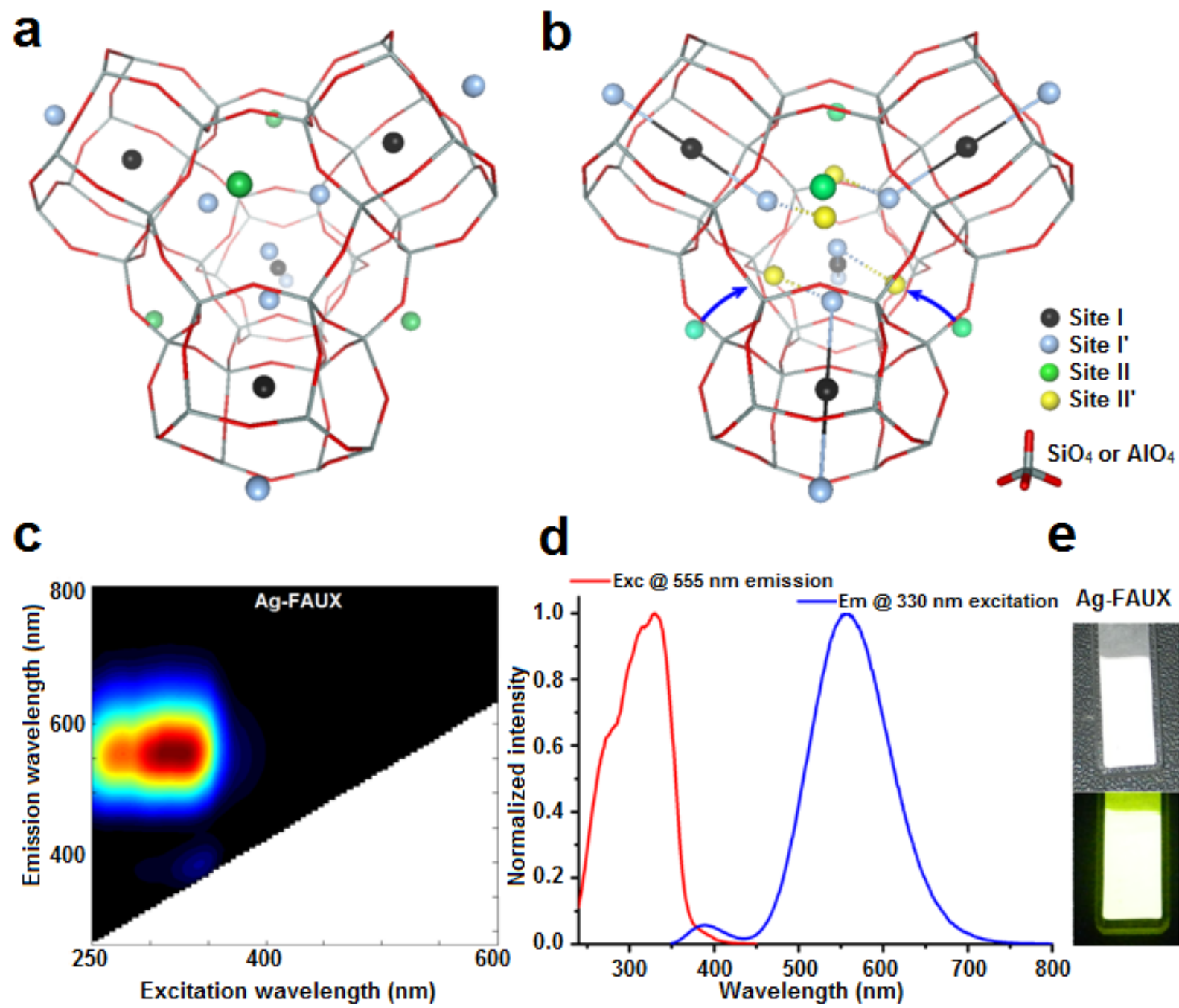

e

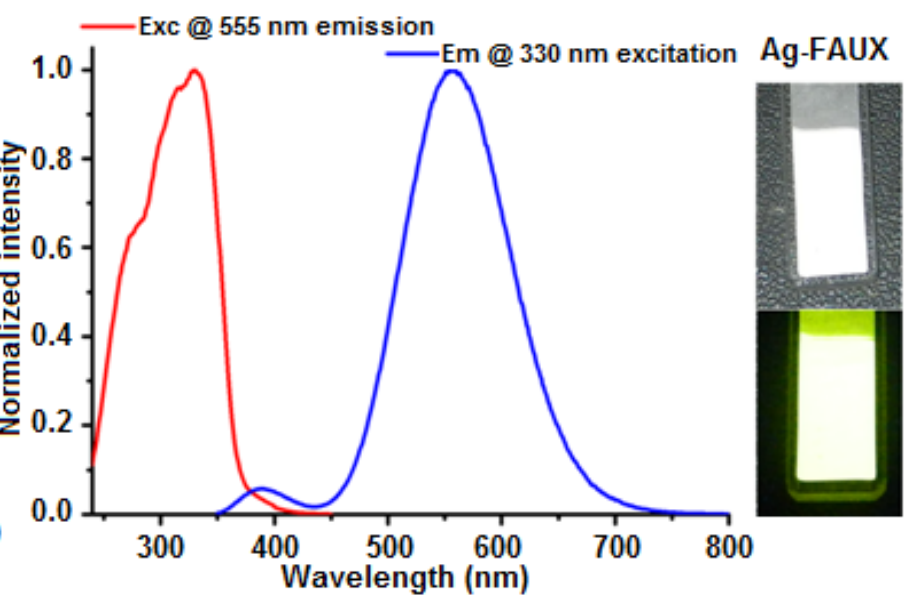

Figure 3. Three-dimensional structural models obtained by HAADF-STEM and XRD analysis for Ag-FAUX zeolites in their non-luminescent (a) and luminescent (b) states. For a better visualization only one sodalite cage with its respective hexagonal connectors (sub-unit cell) is displayed. (c) Two-dimensional excitation-emission plot, (d) excitation-emission profiles and, (e) pictures of heat-treated Ag-FAUX zeolites under day light (top picture) and $366 \mathrm{~nm}$ illumination (bottom picture). 

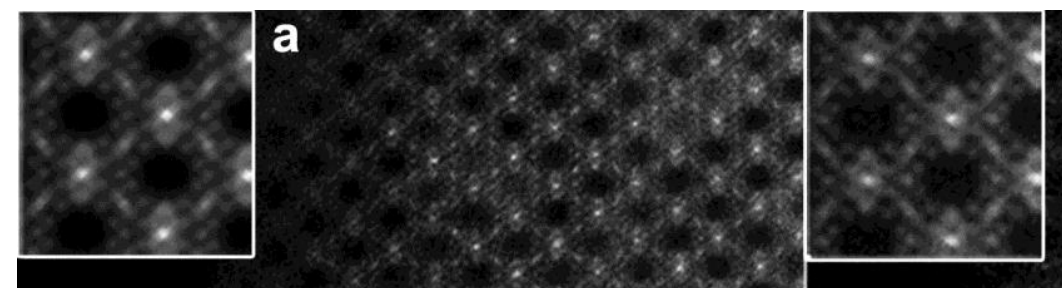

\section{C}

$5 \mathrm{~nm}$

$5 \mathrm{~nm}$

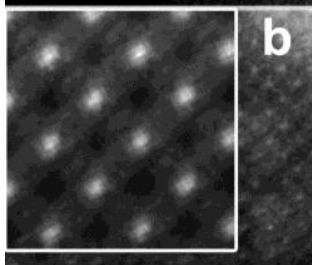

$5 \mathrm{~nm}$

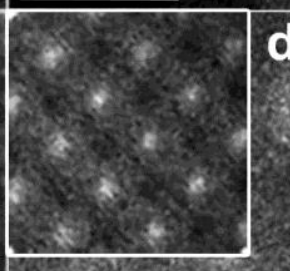

$5 n m$

Figure 4. High resolution HAADF-STEM images of Ag-FAUY zeolites, acquired along a) [110] and b) [100] for the cation-exchanged sample and c) [110] and d) [100] for the heat-treated sample. The insets display the results of the averaging procedure, using 120 templates for (a), 130 for (b), 20 for (c) and 16 for (d), respectively. 


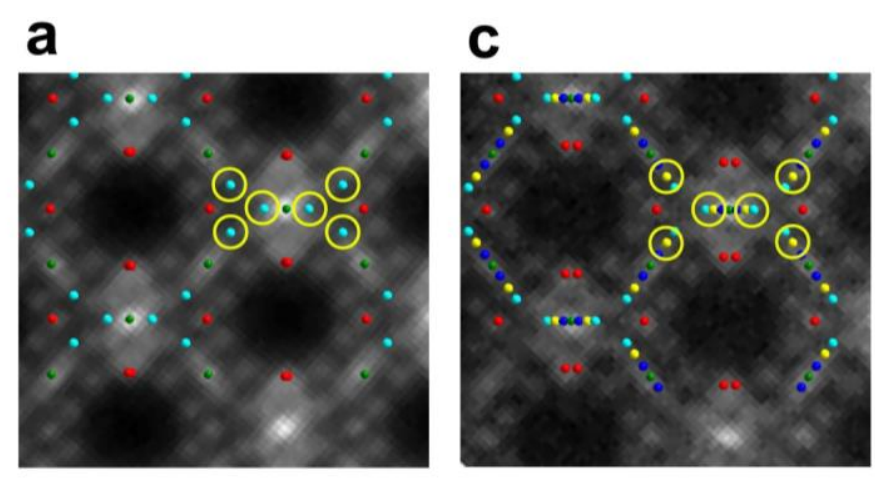

\section{$\underline{1 \mathrm{~nm}}$}

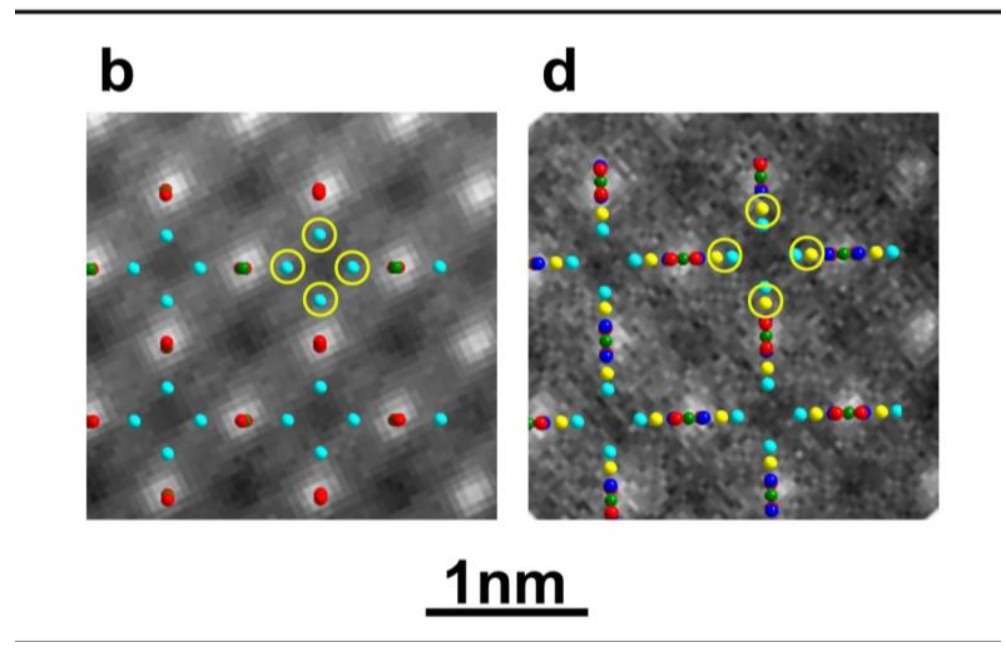

Figure 5. a) and c) Overlay between the averaged HAADF-STEM images and the silver atomic positions obtained from XRD along the [110] zone axis for both the cation-exchanged (a) and heat-treated (c) forms of Ag-FAUY. b) and d) Overlay between the averaged HAADF-STEM images and the silver atomic positions obtained from XRD along the [100] zone axis for both the cation-exchanged (b) and heat-treated (d) forms of Ag-FAUY. The spheres with different colors represent the Ag atoms with different occupancy factors, which are given in Table 2. It can be observed that for both zone axes, no difference can be visualized at the observed intensities, but when comparing the averaged images with the XRD models for both the cation-exchanged and heat-treated forms, inconsistencies were observed, indicated by the yellow circles. For a better visualization, the zeolite framework atoms are not displayed. 

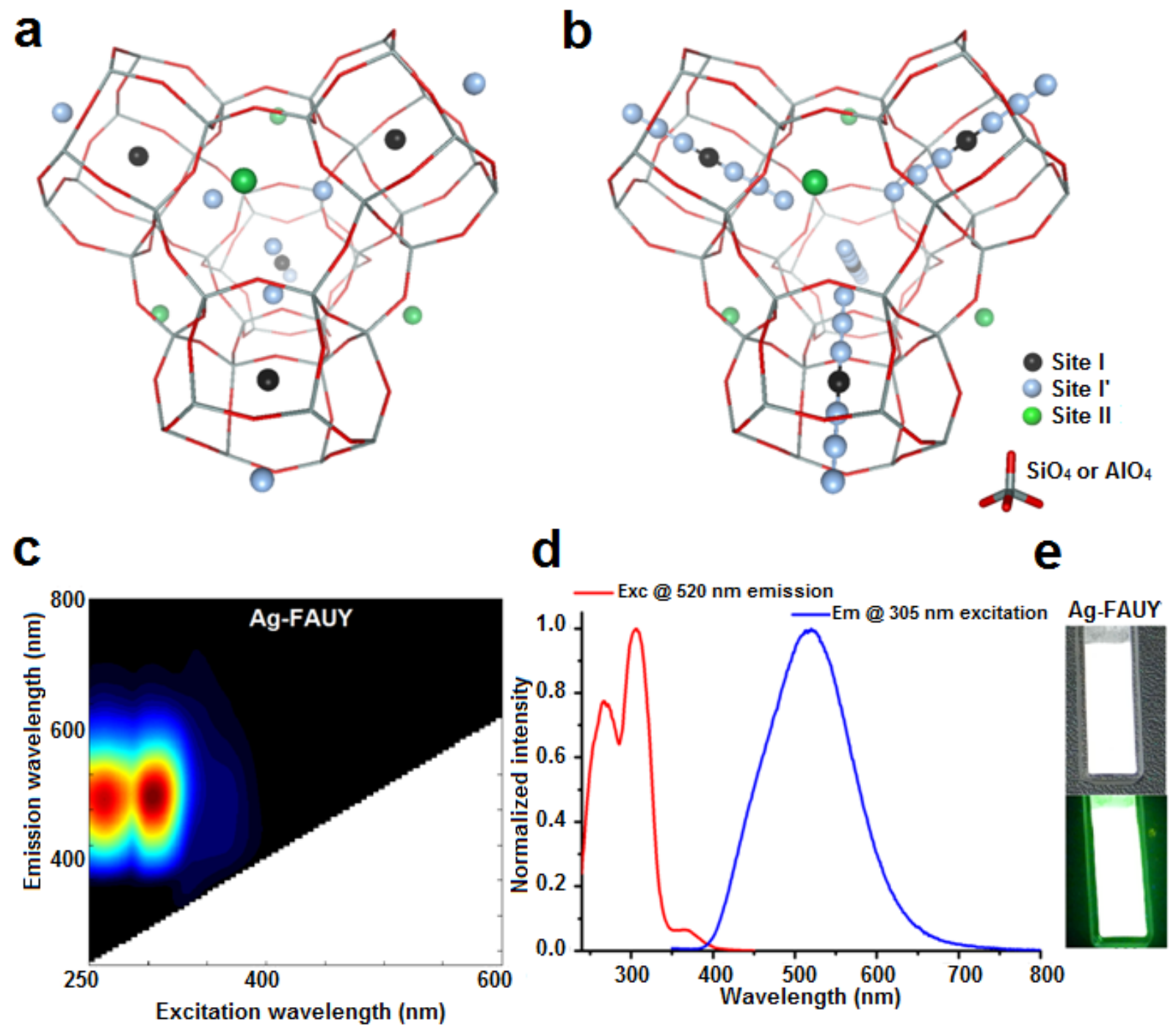

Figure 6. Three-dimensional structural models obtained by HAADF-STEM and XRD analysis for Ag-FAUY zeolites in their non-luminescent (a) and luminescent (b) states. For a better visualization only one sodalite cage with its respective hexagonal connectors (sub-unit cell) is displayed. (c) Two-dimensional excitation-emission plot, (d) excitation-emission profiles and, (e) pictures of heat-treated Ag-FAUY zeolites under day light (top picture) and $366 \mathrm{~nm}$ illumination (bottom picture). 
Table 1. Occupancy factors of the different Ag positions for both cation-exchanged and heattreated forms of Ag-FAUX.

\begin{tabular}{|c|c|c|c|c|c|}
\hline \multicolumn{2}{|c|}{ Ag-FAUX cation-exchanged } & \multicolumn{3}{|c|}{ Ag-FAUX heat-treated } \\
\hline Ag atoms & Site & Occupancy & Ag atoms & Site & Occupancy \\
\hline Ag1 - Cyan & (I') & 0.573 & Ag1 - Cyan & (I') & 0.695 \\
\hline Ag2 - Red & (II) & 0.966 & Ag2 - Red & (II) & 0.598 \\
\hline Ag3 - Green & (I) & 0.971 & Ag3 - Green & (I) & 0.977 \\
\hline & & & Ag4 - Yellow & (II') & 0.319 \\
\hline
\end{tabular}

Table 2. Occupancy factors of the different Ag positions for both cation-exchanged and heattreated forms of Ag-FAUY.

\begin{tabular}{|c|c|c|c|c|c|}
\hline \multicolumn{2}{|c|}{ Ag-FAUY cation-exchanged } & \multicolumn{3}{c|}{ Ag-FAUY heat-treated } \\
\hline Ag atom & Site & Occupancy & Ag atom & Site & Occupancy \\
\hline Ag1 - Cyan & (I') & 0.436 & Ag1 - Cyan & (I') & 0.159 \\
\hline Ag2 - Red & (II) & 0.488 & Ag2 - Red & (II) & 0.480 \\
\hline Ag3 - Green & (I) & 0.125 & Ag3 - Green & (I) & 0.693 \\
\hline & & & Ag4 - Yellow & (I') & 0.137 \\
\hline & & & Ag5 - Blue & (I') & 0.092 \\
\hline
\end{tabular}


Supporting Information. Zeolites characterization, extra HAADF-STEM images and analysis, XRD analysis, additional photoluminescence characterization. This material is available free of charge via the Internet at http://pubs.acs.org.

\section{AUTHOR INFORMATION}

\section{Corresponding Authors}

*E-mail: johan.hofkens@kuleuven.be (J. Hofkens)

*E-mail: sara.bals@uantwerpen.be (S. Bals)

\section{Present Address}

${ }^{¥}$ Skolkovo Institute of Science and Technology, 143025 Moscow, Russia.

\section{Author Contributions}

E. Coutino-Gonzalez prepared the samples and performed the optical and XRD characterization, T. Altantzis performed HAADF-STEM measurements. E. Coutino-Gonzalez, G. Van Tendeloo, M.B.J. Roeffaers, S. Bals and J. Hofkens designed the experiments. All authors contributed to the writing of the manuscript. All authors have given approval to the final version of the manuscript. ${ }^{\ddagger}$ These authors contributed equally.

\section{Notes}

The authors declare no competing financial interest.

\section{ACKNOWLEDGMENT}


The authors gratefully acknowledge financial support from the Belgian Federal government (Belspo through the IAP-VI/27 and IAP-VII/05 programs), the European Union's Seventh Framework Programme (FP7/2007-2013 under grant agreement $\mathrm{n}^{\circ} 310651$ SACS and $\mathrm{n}^{\circ}$ 312483-ESTEEM2), the Flemish government in the form of long-term structural funding “Methusalem" grant METH/08/04 CASAS, the 'Strategisch Initiatief Materialen' SoPPoM program, and the Fund for Scientific Research Flanders (FWO) grant G.0349.12. S. Bals acknowledges funding from ERC Starting Grant COLOURATOMS (335078). The authors thank Prof. S. Van Aert for helpful discussions, Dr. T. De Baerdemaeker for XRD measurements, Mr. B. Dieu for the preparation of graphical material and UOP Antwerp for the kind donation of zeolite samples.

\section{REFERENCES}

1) Xu, Z.; Xiao, F.S.; Purnell, S.K.; Alexeev, O.; Kawi, S.; Deutsch, S.E.; Gates, B.C. SizeDependent Catalytic Activity of Supported Metal-Clusters. Nature 1994, 372, 346-348.

2) Corma, A.; Concepcion, P.; Boronat, M.; Sabater, M.J.; Navas, J.; Yacaman, M.J.; Larios, E.; Posadas, A.; Lopez-Quintela, M.A.; Bucetta, D.; Mendoza, E.; Guilera, G.; Mayoral, A. Exceptional Oxidation Activity With Size-Controlled Supported Gold Clusters of Low Atomicity. Nat. Chem. 2013, 5, 775-781.

3) Desireddy, A.; Conn, B.E.; Guo, J.; Yoon, B.; Barnett, R.N.; Monahan, B.M.; Kirschbaum, K.; Griffith, W.P.; Whetten, R.L.; Landman, U.; Bigioni, T.P. Ultrastable Silver Nanoparticles. Nature 2013, 501, 399-402. 
4) De Cremer, G.; Sels, B.F.; Hotta, J.; Roeffaers, M.B.J.; Bartholomeeusen, E.; CoutinoGonzalez, E.; Valtchev, V.; De Vos, D.E.; Vosch, T.; Hofkens, J. Optical Encoding of Silver Zeolite Microcarriers. Adv. Mater. 2010, 22, 957-960.

5) Royon, A.; Bourhis, K.; Bellec, M.; Papon, G.; Bousquet, B.; Deshayes, Y.; Cardinal, T.; Canioni, L. Silver Clusters Embedded in Glass as a Perennial High Capacity Optical Recording Medium. Adv. Mater. 2010, 22, 5282-5286.

6) Stamplecoskie, K.G.; Chen, Y.-S.; Kamat, P.V. Excited-State Behavior of Luminescent Glutatione-Protected Gold Clusters. J. Phys. Chem. C 2014, 118, 1370-1376.

7) Goswami, N.; Yao, Q.; Luo, Z.; Li, J.; Chen, T.; Xie, J. Luminescent Metal Nanoclusters with Aggregation-Induced Emission. J. Phys. Chem. Lett. 2016, 7, 962-975.

8) Lu, Y.; Chen, Y. Sub-Nanometre Sized Metal Clusters: From Synthetic Challenges to the Unique Property Discoveries. Chem. Soc. Rev. 2012, 41, 3594-3623.

9) Choi, S.; Dickson, R.M.; Yu, J.H. Developing Luminescent Silver Nanodots for Biological Applications. Chem. Soc. Rev. 2012, 41, 1867-1891.

10) Aydin, C.; Lu, J.; Shirai, M.; Browning, N.D.; Gates, B.C. Ir-6 Clusters Compartmentalized in the Supercages of Zeolite NaY: Direct Imaging of a Catalyst with Aberration-Corrected Scanning Transmission Electron Microscopy. ACS Catal. 2011, 1, 16131620.

11) Kuge, K.; Calzaferri, G. Gold-Loaded Zeolite A. Microporous Mesoporous Mater. 2003, $66,15-20$. 
12) Mayoral, A.; Readman, J.E.; Anderson, P.A. Aberration-Corrected STEM Analysis of a Cubic Cd Array Encapsulated in Zeolite A. J. Phys. Chem. C 2013, 117, 24485-24489.

13) Patterson, H.H.; Gomez, R.S.; Lu, H.; Yson, R.L. Nanoclusters of Silver Doped in Zeolites as Photocatalysts. Catal. Today 2007, 120, 168-173.

14) Hutson, N.D.; Rege, S.U.; Yang, R.T. Mixed Cation Zeolites: LixAgy-X as Superior Adsorbent for Air Separation. AIChE J. 1999, 45, 724-734.

15) Seuong, D.; Lee, Y.; Cynn, H.; Park, C.; Choi, K.Y.; Blom, D.A.; Evans, W.J.; Kao, C.C.; Vogt, T.; Lee, Y. Irreversible Xenon Insertion Into a Small-Pore Zeolite at Moderate Pressures and Temperatures. Nat. Chem. 2013, 6, 835-839.

16) De Cremer, G.; Coutino-Gonzalez, E.; Roeffaers, M.B.J.; Moens, B.; Ollevier, J.; Auweraer, M.; Schoonheydt, R.; Jacobs, P.A.; De Schryver, F.C.; Hofkens, J.; De Vos, D.E.; Sels, B.F.; Vosch, T. Characterization of Fluorescence in Heat-Treated Silver-Exchanged Zeolites. J. Am. Chem. Soc. 2009, 131, 3049-3056.

17) Coutino-Gonzalez, E.; Roeffaers, M.B.J.; Dieu, B.; De Cremer, G.; Leyre, S.; Hanselaer, P.; Fyen, W.; Sels, B.F.; Hofkens, J. Determination and Optimization of the Luminescence External Quantum Efficiency of Silver-Clusters Zeolite Composites. J. Phys. Chem. C 2013, 117, 6998-7004.

18) Coutino-Gonzalez, E.; Baekelant, W.; Grandjean D.; Roeffaers, M.B.J.; Fron, E.; M.S. Aghakhani.; N. Bovet.; M. van der Auweraer.; P. Lievens.; T. Vosch.; Sels, B.F.; Hofkens, J. Thermally Activated LTA(Li)-Ag Zeolites With Water-Responsive Photoluminescence Properties. J. Mater. Chem. C 2015, 3, 11857-11867. 
19) Fenwick, O.; Coutino-Gonzalez, E.; Grandjean D.; Baekelant, W.; Richard, F.; Bonacchi, S.; De Vos, D.; Lievens, P.; Roeffaers, M.B.J.; Hofkens, J.; Samori, P. Tuning the Energetics and Tailoring the Optical Properties of Silver Clusters Confined in Zeolites. Nat. Mater. 2016, DOI: $10.1038 /$ nmat4652.

20) Coutino-Gonzalez, E.; Grandjean D.; Roeffaers, M.B.J.; Kvashnina, K.; Fron, E.; Dieu, B.; De Cremer, G.; Lievens, P.; Sels, B.F.; Hofkens, J. X-ray Irradiation-Induced Formation of Luminescent Silver Clusters in Nanoporous Materials. Chem. Commun. 2014, 50, 1350-1352.

21) Mayoral, A.; Carey, T.; Anderson, P.A.; Lubk, A.; Diaz, I. Atomic Resolution Analysis of Silver Ion-Exchanged Zeolite A. Angew. Chem., Int. Ed. 2011, 50, 11230-11233.

22) Kim, C.W.; Heo, N.H.; Seff, K. Framework Sites Preferred by Aluminum in Zeolite ZSM5. Structure of a Fully Dehydrated, Fully CS+-Exchanged ZSM-5 Crystal (MFI, Si/Al=24). J. Phys. Chem. C 2011, 115, 24823-24838.

23) Frising, T.; Leflaive, P. Extraframework Cation Distributions in $X$ and $Y$ Faujasite Zeolites: A Review. Microporous Mesoporous Mater. 2008, 114, 27-63.

24) Gellens, L.R.; Mortier, W.J.; Uytterhoeven, J.B. On the Nature of the Charged Silver Clusters in Zeolites of type A, X and Y. Zeolites 1981, 1, 11-18.

25) Lee, H.S.; Kim, Y.; Seff, K. Weak Ag+-Ag+ Bonding in Zeolite X. Crystal Structures of $\mathrm{Ag}_{92} \mathrm{Si}_{100} \mathrm{Al}_{92} \mathrm{O}_{384}$ Hydrated and Fully Dehydrated in Flowing Oxygen. Microporous Mesoporous Mater. 2000, 41, 49-59. 
26) Kim, S.Y.; Kim, Y.; Seff, K. Two Crystal Structures of Fully Dehydrated, Fully Ag+Exchanged Zeolite X. Dehydration in Oxygen Prevents Ag+ Reduction. Without Oxygen, Ag8(n+) (T-d) and Cyclo-Ag-4(m+) (near S-4) Form. J. Phys. Chem. B 2003, 107, 6938-6945.

27) Mayoral, A.; Carey, T.; Anderson, P.A., Diaz, I. Atomic Resolution Analysis of Porous Solids: A Detailed Study of Silver Ion-Exchanged Zeolite A. Microporous Mesoporous Mater. 2013, 166, 117-122.

28) Sasaki, Y.; Suzuki, T. Formation of Ag Clusters by Electron Beam Irradiation of AgZeolites. Mater. Trans. 2009, 50, 1050-1053.

29) Devijver, P.A.; Kittler, J. Pattern Recognition: A Statistical Approach. Prentice-Hall International, UK, 1982; pp: 232-238.

30) Pennycook, S.J. Z-Contrast Transmission Electron-Microscopy - Direct Atomic Imaging of Materials. Annu. Rev. Mater. Sci. 1992, 22, 171-195.

31) Alonso, J.A.; Lopez, M.J.; Molina, L.M.; Duque, F.; Mananes, A. Conditions for the SelfAssembling of Cluster Materials. Nanotechnology. 2002, 13, 253-257.

32) Diez, I.; Ras, R.H.A. Fluorescent Silver Nanoclusters. Nanoscale. 2011, 3, 1963-1970.

33) Seifert, R.; Rytz, R.; Calzaferri, G. Colors of Ag+-Exchanged Zeolite A. J. Phys. Chem. A 2000, 104, 7473-7483.

34) Seifert, R.; Kunzmann, A.; Calzaferri, G. The Yellow Color of Silver-Containing Zeolite A. Angew. Chem., Int. Ed. 1998, 37, 1522-1524. 
35) Petricek, V.; Dusek, M.; Palatinus, L. Crystallographic Computing System JANA2006: General Features. Z. Kristallogr. 2014, 229, 345-352. 